\title{
PRODUTIVIDADE E EFICIÊNCIA DE USO DE NITROGÊNIO NO MILHO SAFRINHA
}

\section{YIELD AND THE NITROGEN USE EFFICIENCY IN MAIZE CROP OFF-SEASON}

\author{
Artur Pessim ${ }^{1}$, Leonardo Oliveira Marciano ${ }^{1}$ e Rogério Farinelli ${ }^{1}$ \\ ${ }^{1}$ Centro Universitário da Fundação Educacional de Barretos (UNIFEB), Avenida Professor Frade Monte, 389. \\ CEP 14783-226, Barretos, SP.
}

\section{Resumo}

$\mathrm{O}$ nitrogênio $(\mathrm{N})$ é nutriente exigido em maior quantidade pela cultura do milho, sendo o que mais frequentemente limita a produtividade de grãos. Contudo, a definição da dose, da melhor época de aplicação em cobertura, bem como a eficiência de uso do $\mathrm{N}$ deve ser mais bem estabelecido em cada sistema de produção. Assim, o objetivo do trabalho foi avaliar características agronômicas e eficiência de uso de $\mathrm{N}$ na safrinha, em função de doses e épocas de aplicação em cobertura. O delineamento experimental empregado foi o de blocos ao acaso, em esquema fatorial $2 \times 4$, com quatro repetições. Os tratamentos foram constituídos por duas épocas de aplicação de $\mathrm{N}$ (estádio V5 e estádio V10) e quatro doses em cobertura $\left(0,30,60\right.$ e $\left.90 \mathrm{~kg} \mathrm{ha}^{-1}\right)$, fonte ureia. Os resultados demonstraram influência das doses no teor de $\mathrm{N}$ foliar e no número de grãos por espiga. A produtividade foi superior $50 \mathrm{~kg} \mathrm{ha}^{-1} \mathrm{de} \mathrm{N}$, sendo significativa com a aplicação no estádio V10. Tal fato também foi comprovado pela eficiência agronômica, agrofisiológica e de recuperação e onde os maiores resultados foram obtidos no estádio $\mathrm{V} 10$, com aplicação de $30 \mathrm{~kg} \mathrm{ha}^{-1} \mathrm{de} \mathrm{N}$.

Palavras-chave: Zea mays, adubação nitrogenada, características agronômicas, eficiência agronômica.

\begin{abstract}
Nitrogen $(\mathrm{N})$ is a nutrient demanded by the maize crop, which most often limits grains yield. However, the definition of the dose, of the best time of topdressing application, as well as the nitrogen use efficiency should be better established, especially in each crop system. Therefore, this work aimed to evaluate agronomic characteristics and efficiency of nitrogen in maize grown in the second crop, as a function of application times and nitrogen topdressing. The experimental design was randomized blocks, factorial $2 \mathrm{x}$ 4 with four replications. The treatments consisted of two application times (V5 and V10) and four N doses $\left(0,30,60\right.$, and $\left.90 \mathrm{~kg} \mathrm{ha}^{-1}\right)$, source of urea. The results showed that there was an influence of doses in leaf $\mathrm{N}$ content and the number of grains per ear. The yield was higher with the application of $50 \mathrm{~kg} \mathrm{ha}^{-1}$ of $\mathrm{N}$, being significant with the application in the V10 stage. This fact was also confirmed by the agronomic, agrophysiological, and recovery efficiency, where the highest results were obtained in the V10 stage, with the application of $30 \mathrm{~kg} \mathrm{ha}^{-1}$ of nitrogen topdressing.
\end{abstract}

Keywords: Zea mays, nitrogen fertilization, agronomic characteristics, agronomic efficiency.

Autor correspondente: Rogerio Farinelli; Centro Universitário da Fundação Educacional de Barretos (UNIFEB), Avenida Professor Frade Monte, 389. CEP 14783-226, Barretos, SP (16) 997099956. E-mail: rogerio.farinelli@unifeb.edu.br

Recebido para publicação: $16 /$ maio/2021

Aceito para publicação: 30/nov/2021

https://doi.org/10.4322/1980-0029.122021 


\section{Introdução}

O milho é uma cultura extremamente eficiente na conversão de energia luminosa em biomassa, apresentando elevada exigência nutricional, com destaque para o nitrogênio $(\mathrm{N})$, o qual é exigido em maior quantidade pela cultura, sendo o que mais frequentemente limita a produtividade de grãos, pois exerce importante função nos processos bioquímicos e fisiológicos da planta (Fancelli \& Dourado, 2008).

Algumas estratégias podem ser tomadas com intuito de aumentar a eficiência no uso de $\mathrm{N}$, tais como a redução das doses de adubos para níveis que sejam produtivos e seguros, a utilização de leguminosas em rotação e a fixação biológica de $\mathrm{N}$ em gramíneas (Carvalho et al., 2012). Dentro deste contexto, ainda podem ser elencadas a produtividade atingida, as condições climáticas, fertilidade do solo, adubação realizada e demais tratos culturais.

No caso do milho de segunda safra (safrinha), a adubação de cobertura pode ser primordial para obtenção de maiores rentabilidades pelos produtores, porém a reduzida disponibilidade hídrica em comparação à primeira safra pode ser limitante à época de aplicação do N (Góes et al., 2012). A recomendação da adubação nitrogenada de cobertura para o milho referente a uma determinada região é complexa, devido às transformações que ocorrem no solo relacionado à sua alta mobilidade e aos fatores que influenciam no seu aproveitamento pelas plantas.

Resultados experimentais mostraram respostas generalizadas do milho à adubação nitrogenada, onde aproximadamente $70 \%$ a $90 \%$ dos ensaios de adubação realizados em campo, no Brasil, são responsivos à aplicação de nitrogênio (Góes et al., 2012). Pesquisas realizadas nos últimos anos demonstraram que o potencial produtivo da cultura está relacionado à aplicação de doses de $\mathrm{N}$ em cobertura entre 60 e $100 \mathrm{~kg} \mathrm{ha}^{-1}$, para cultivo em sequeiro, e 120-160 $\mathrm{kg} \mathrm{ha}^{-1}$ de $\mathrm{N}$, para cultivo irrigado (Souza et al., 2011; Farinelli \& Lemos, 2012, Soratto et al., 2012; Duarte \& Kappes, 2013).

Duarte \& Kappes (2013), estudando a resposta do milho safrinha à adubação nitrogenada ( 0 , 30,60 e $\left.90 \mathrm{~kg} \mathrm{ha}^{-1}\right)$, verificaram que, quando o $\mathrm{N}$ é aplicado até $39 \mathrm{~kg} \mathrm{ha}^{-1}$ na semeadura, a produtividade acima de $6.500 \mathrm{~kg} \mathrm{ha}^{-1}$ é alcançada com aproximadamente $65 \mathrm{~kg} \mathrm{ha}^{-1} \mathrm{de} \mathrm{N}$ em cobertura. Quando o N é fornecido apenas em cobertura, os resultados são inferiores. Nesse estudo, as doses de $\mathrm{N}$ em cobertura foram aplicadas no estádio V5.

Segundo o trabalho realizado por Gazola et al. (2014), o teor de $\mathrm{N}$ foliar e a produtividade de grãos apresentaram ajuste a uma equação linear e quadrática, respectivamente, em resposta às doses de $\mathrm{N}$ aplicadas em cobertura $(0,60,120 \mathrm{e}$ $\left.180 \mathrm{~kg} \mathrm{ha}^{-1}\right)$. A máxima produtividade ocorreu por meio da dose de $149,5 \mathrm{~kg} \mathrm{ha}^{-1}$, com $3.107 \mathrm{~kg}$ ha $^{-1}$, acréscimo de $46 \%$ em relação ao tratamento controle.

Sichocki et al. (2014) alcançaram efeito positivo na produtividade de grãos de milho safrinha pela elevação das doses de $\mathrm{N}$ em cobertura $(0 \mathrm{a}$ $150 \mathrm{~kg} \mathrm{ha}^{-1}$ ) aplicadas no estádio V6, com valor ultrapassando $6.000 \mathrm{~kg} \mathrm{ha}^{-1}$. Resultado semelhante foi verificado por Fuentes et al. (2018) com acréscimo significativo em relação às doses de $\mathrm{N}$ em cobertura $\left(0,30,60,90\right.$ e $\left.120 \mathrm{~kg} \mathrm{ha}^{-1}\right)$, no estádio V5. Também houve efeito positivo para demais características agronômicas, como massa de 1.000 grãos, número de grãos por espiga, e teores de $\mathrm{N}$ na folha e nos grãos.

Em relação à época de aplicação, os melhores resultados para produtividade foram obtidos quando foi realizada a adubação em V2, V4 e V8, sendo que a dose de $\mathrm{N}$ em cobertura foi de $100 \mathrm{~kg} \mathrm{ha}^{-1}$ (Gott et al., 2014).

O estudo da adubação nitrogenada em cobertura na cultura do milho é uma prática importante e inserida nos aspectos de nutrição mineral, permitindo minimizar os custos de produção. No entanto, a definição da dose adequada, da melhor época de aplicação, bem como a eficiência de uso do nutriente, deve ser precisamente estabelecida, principalmente em cada sistema de produção, pois depende, dentre outos fatores já citados anteriormente, também do genótipo (Carvalho et al., 2012). De acordo com os autores, as cultivares de milho apresentaram características distintas quanto à eficiência no uso e absorção de nitrogênio em diferentes ambientes quanto à disponibilidde de $\mathrm{N}$.

Os híbridos são menos eficientes no uso do $\mathrm{N}$ em altos níveis do suplemento nitrogenado. Além disso, a eficiência do uso de $\mathrm{N}$ diminiu em relação ao aumento de doses aplicadas, em vista de o suprimento de $\mathrm{N}$ exceder as necessidades da cultura (Fernandes et al., 2005).

O objetivo do trabalho foi avaliar o desempenho da cultura do milho safrinha quanto à produtividade e eficiência de uso de nitrogênio em função da 
adubação nitrogenada de cobertura e épocas de aplicação.

\section{Material e Métodos}

O experimento foi desenvolvido no UNIFEB - Centro Universitário da Fundação Educacional de Barretos, com coordenadas geográficas de latitude $20^{\circ} 33^{\prime}$ Sul e longitude $48^{\circ} 34^{\prime}$ ' Oeste, a uma altitude de 530 metros. O clima da região, segundo a classificação de Köppen, é Aw, ou seja, com inverno seco e moderado, e verão quente e chuvoso.

O solo da área foi classificado como Latossolo Vermelho distroférrico, textura argilosa, sendo que a caracterização química realizada antes da instalação do experimento revelou os seguintes atributos $(0-20 \mathrm{~cm}): \mathrm{pH}\left(\mathrm{CaCl}_{2}\right)$ de 5,$0 ; 22,0 \mathrm{~g} \mathrm{dm}^{-3}$ de MO; 10,0 mg dm ${ }^{-3}$ de P; 1,8 mmol $_{\mathrm{c}} \mathrm{dm}^{-3} \mathrm{de} \mathrm{K}$; 19,0 $\mathrm{mmol}_{\mathrm{c}} \mathrm{dm}^{-3}$ de $\mathrm{Ca} ; 7,0 \mathrm{mmol}_{\mathrm{c}} \mathrm{dm}^{-3}$ de $\mathrm{Mg}$; 24,7 $\mathrm{mmol}_{\mathrm{c}} \mathrm{dm}^{-3}$ de $\mathrm{H}+\mathrm{Al} ; 27,8 \mathrm{mmol}_{\mathrm{c}} \mathrm{dm}^{-3}$ de $\mathrm{SB} ; 52,5 \mathrm{mmol}_{\mathrm{c}} \mathrm{dm}^{-3}$ de CTC e saturação por bases de $52,9 \%$.

Aárea experimental foi cultivada anteriormente com milho de primeira safra, sendo colhido para silagem, depois realizado o preparo de solo convencional. Na adubação de semeadura foram aplicados 18, 63 e $36 \mathrm{~kg} \mathrm{ha}^{-1}$ de N, $\mathrm{P}_{2} \mathrm{O}_{5}$ e $\mathrm{K}_{2} \mathrm{O}$, respectivamente, seguindo as recomendações de Cantarella \& Duarte (1997), para uma produtividade estimada de 4.000 a $6.000 \mathrm{~kg} \mathrm{ha}^{-1}$ de grãos.

A semeadura foi realizada mecanicamente em 28/02/2013 no período de cultivo de segunda safra (safrinha) para a região Norte/Nordeste do Estado de São Paulo, utilizando o espaçamento entrelinhas de $0,50 \mathrm{~m}$, com densidade populacional de 56.000 plantas ha $^{-1}$.

O híbrido utilizado foi o AG 8088 VT PRO2, que possui dois eventos de tecnologia transgênica, sendo a primeira com característica de resistência a espécies de insetos (tecnologia Bt $($ ) da ordem Lepidoptera, e a segunda tecnologia de resistência ao glifosato (tecnologia $R R \AA$ ), permitindo um controle mais eficaz de plantas daninhas. A cultivar apresenta característica de híbrido simples, ciclo precoce (870 graus-dias), recomendada para o cultivo de safra primavera-verão e safrinha, possui grãos alaranjados e do tipo duro, com finalidade de produção de grãos e silagem, e recomendados para a região Sudeste (Cruz et al., 2012).

O delineamento experimental empregado foi o de blocos ao acaso, em esquema fatorial $2 \mathrm{x}$
4, com quatro repetições. Os tratamentos foram constituídos por duas épocas de aplicação de $\mathrm{N}$, representadas pelo estádio V5 e pelo estádio V10 (caracterizados pelo desdobramento da quinta e décima folha, respectivamente) e por quatro doses de nitrogênio em cobertura $\left(0,30,60\right.$ e $\left.90 \mathrm{~kg} \mathrm{ha}^{-1}\right)$, fonte ureia. As parcelas experimentais foram constituídas de seis linhas de $5 \mathrm{~m}$, no espaçamento de $0,50 \mathrm{~m}$, considerando como área útil apenas as duas linhas centrais. A aplicação das doses de $\mathrm{N}$ em cobertura foi realizada em filete contínuo a $10 \mathrm{~cm}$ de distância das plantas de milho.

Os tratamentos fitossanitários, como manejo de plantas daninhas e insetos-praga, foram empregados utilizando-se produtos recomendados à cultura.

No estádio de florescimento feminino (R1) foram coletadas em dez plantas ao acaso por parcela o terço central de dez folhas opostas e abaixo à primeira espiga (Coelho et al., 2002), e após a secagem em estufa de circulação forçada de ar a $60-70^{\circ} \mathrm{C}$ por 72 horas, as folhas foram moídas em moinho tipo Wiley, sendo então determinado o teor de $\mathrm{N}$ segundo metodologia descrita por Malavolta et al. (1997)

A colheita foi realizada manualmente, na área útil de cada parcela, tendo sido colhidas todas as espigas com palha. Posteriormente, em dez espigas por parcela avaliou-se o número de grãos por espiga. A massa de 1.000 grãos foi realizada por meio da coleta de duas amostras e posterior pesagem, sendo calculada em $13 \%$ de base úmida. A produtividade de grãos foi obtida por meio da massa de grãos, determinando-se o teor de água calculado em 13\% de base úmida (Brasil, 2009).

De acordo com as metodologias propostas por Fageria \& Baligar (2005), também foram determinados os modos de eficiência de uso de nitrogênio. A eficiência agronômica (EA) foi calculada utilizando a fórmula: $\mathrm{EA}=\left(\mathrm{PG}_{\mathrm{cf}}-\right.$ $\left.\mathrm{PG}_{\mathrm{sf}}\right) /\left(\mathrm{QN}_{\mathrm{a}}\right)$, expressa em $\mathrm{kg} \mathrm{kg}^{-1}$, em que $\mathrm{PG}_{\mathrm{cf}}$ é a produção de grãos com fertilizante nitrogenado; $\mathrm{PG}_{\mathrm{sf}}$ é a produção de grãos sem fertilizante nitrogenado e $\mathrm{QN}_{\mathrm{a}}$ é a quantidade de nitrogênio aplicado em $\mathrm{kg}$. A eficiência agrofisiológica $(\mathrm{EAF})$ foi calculada mediante a fórmula: $\mathrm{EAF}=$ $\left(\mathrm{PG}_{\mathrm{cf}}-\mathrm{PG}_{\mathrm{sf}}\right) /\left(\mathrm{AC}_{\mathrm{cf}}-\mathrm{AC}_{\mathrm{sf}}\right)$, expressa em kg kg-1, em que $\mathrm{AC}_{\mathrm{cf}}$ é a quantidade de $\mathrm{N}$ acumulado na parte aérea e nos grãos com fertilizante nitrogenado e $\mathrm{ACs}_{\mathrm{f}}$ é a quantidade de $\mathrm{N}$ acumulado na parte aérea e nos grãos sem fertilizante nitrogenado. $\mathrm{E}$ a eficiência de recuperação (ER) foi determinada 
pela equação: $\mathrm{ER}=\left(\mathrm{AC}_{\mathrm{cf}}-\mathrm{AC}_{\mathrm{sf}}\right) /\left(\mathrm{QN}_{\mathrm{a}}\right) \times 100$, expressa em porcentagem.

Os dados foram submetidos à análise de variância, empregando-se o teste F. Para a comparação dos valores das épocas de aplicação foi utilizado o teste de Tukey a $5 \%$ de probabilidade e análise de regressão polinomial para a comparação dos valores das doses de $\mathrm{N}$ em cobertura, bem como das interações significativas entre épocas de aplicação e doses de $\mathrm{N}$ em cobertura. As análises estatísticas foram realizadas com o auxílio do software Sisvar 5.3 (Ferreira, 2011).

\section{Resultados e Discussão}

O teor de nitrogênio foliar é um parâmetro significativo para detectar a quantidade de $\mathrm{N}$ absorvida pela planta. De acordo com a Tabela 1, verifica-se influência da adubação nitrogenada sobre o teor de $\mathrm{N}$ nas folhas $(\mathrm{y}=0,086 \mathrm{x}+22,23)$. Segundo Fancelli \& Dourado (2008), o nível adequado do teor de nitrogênio na folha de milho para um desenvolvimento também satisfatório está situado na faixa de 27,5 a $32,5 \mathrm{~g} \mathrm{~kg}^{-1}$ Portanto, para as doses de 60 e $90 \mathrm{~kg} \mathrm{ha}^{-1}$ de $\mathrm{N}$ houve melhores resultados, proporcionando uma boa nutrição do elemento às plantas. É válido ressaltar que, na testemunha e na menor dose, as plantas apresentaram no início do desenvolvimento sintomas de deficiência do nutriente. Este resultado positivo da adubação de cobertura também foi obtido por
Gazola et al. (2014), tendo desempenho semelhante quanto aos valores com doses de 0 a $180 \mathrm{~kg} \mathrm{ha}^{-1}$ de $\mathrm{N}$, o que pode ser explicado pelas condições edafoclimáticas do cultivo.

Para o número de grãos por espiga também foi obtido efeito das doses de $\mathrm{N}$ em cobertura $(y=0,58 x+310)($ Tabela 1), corroborando com Sichocki et al. (2014), porém estes autores empregaram quantidades de até $150 \mathrm{~kg} \mathrm{ha}^{-1}$ de $\mathrm{N}$ em cobertura, aplicadas no estádio V6. A ausência de valores significativos quanto às duas épocas de aplicação se assemelham aos resultados de Gott et al. (2014), onde não houve diferenças com a adubação nitrogenada nos estádios V2, V4, V6 e V8, mas foi incrementado em relação à não aplicação do nutriente.

A massa de 1.000 grãos é característica influenciada pela disponibilidade de nutrientes. No entanto, nota-se que isso não significou aumento considerável no respectivo trabalho (Tabela 1), corroborando Góes et al. (2012), com doses de 0 a $80 \mathrm{~kg} \mathrm{ha}^{-1}$ de $\mathrm{N}$ em cobertura, bem como a Gott et al. (2014) em diferentes épocas de aplicação (V2 a V8). A massa de grãos é uma característica também influenciada pelo genótipo e pelas condições climáticas durante a fase de enchimento de grãos. Durante o desenvolvimento do respectivo trabalho, ocorreu um período de déficit hídrico de aproximadamente 40 dias, que coincidiu principalmente com a fase de enchimento

Tabela 1. Teor de $\mathrm{N}$ foliar, número de grãos por espiga e massa de 1.000 grãos da cultura do milho safrinha em função de doses de N em cobertura e épocas de aplicação. Barretos, SP, 2013.

\begin{tabular}{lccc}
\hline \multicolumn{1}{c}{ Tratamentos } & $\begin{array}{c}\text { Teor de N Foliar } \\
\left(\mathbf{g ~ k g}^{-1}\right)\end{array}$ & $\begin{array}{c}\text { Grãos por Espiga } \\
\left(\mathbf{n}^{\mathbf{0}}\right)\end{array}$ & $\begin{array}{c}\text { Massa de 1.000 grãos } \\
(\mathbf{g})\end{array}$ \\
\hline Doses de N em cobertura (D) & & & \\
0 & $22,0^{(1)}$ & $311^{(2)}$ & 190 \\
30 & 24,6 & 324 & 194 \\
60 & 28,5 & 349 & 198 \\
90 & 29,4 & 361 & 201 \\
Teste F & $9,38^{*}$ & $5,09^{* *}$ & $2,34^{\text {ns }}$ \\
Épocas de aplicação (E) & & & \\
V5 & 26,4 & 335 & 20,3 \\
V10 & 25,9 & 338 & 19,9 \\
Teste F & $0,20^{\text {ns }}$ & $0,74^{\text {ns }}$ & $0,88^{\text {ns }}$ \\
D x E & & & \\
Teste F & $0,28^{\text {ns }}$ & $0,29^{\text {ns }}$ & $0,95^{\text {ns }}$ \\
Média & 26,1 & 336 & 201 \\
CV\% & 12,13 & 8,51 & 6,06 \\
\hline (1) $y=0,086 x+22,23$ e R2 $=0,95^{* * ;}$ (2) $y=0,58 x+310$ e R $R^{2}=0,97^{* *} ;$ ns Não significativo. * e ** (Significativo a $5 \%$ e a \\
1\% de probabilidade, respectivamente).
\end{tabular}


de grãos (estádios R3 e R4), o que pode ter afetado este componente da produção.

A produtividade máxima de $3.818 \mathrm{~kg} \mathrm{ha}^{-1} \mathrm{de}$ grãos foi atingida com dose próxima a $50 \mathrm{~kg} \mathrm{ha}^{-1}$, sendo mais significativo quando o $\mathrm{N}$ foi aplicado no estádio V10 (Figura 1). A resposta a favor desta época de aplicação foi semelhante à obtida por Kappes et al. (2009). Em se tratando de milho safrinha, eventos climáticos adversos muitas vezes são a causa de resultados inferiores de produtividade, interferindo negativamente no efeito da adubação. Fato que ocorreu com Góes et al. (2012), em que não houve resposta significativa ao nitrogênio fornecido em cobertura, tampouco quanto às épocas de aplicação do nutriente (V2 ao V8), devido aos baixos índices pluviométricos.

Duarte \& Kappes (2013) também obtiveram resposta quadrática em função da aplicação de $\mathrm{N} \mathrm{em}$ cobertura com a dose estimada de aproximadamente $65 \mathrm{~kg} \mathrm{ha}^{-1}$, com a adubação aplicada no estádio V5. Enquanto que Souza et al. (2011) e Sichocki et al. (2014) verificaram acréscimo na produtividade de grãos de milho safrinha pela elevação das doses de $\mathrm{N}$ em cobertura $\left(0,30,60,90,120\right.$ e $\left.150 \mathrm{~kg} \mathrm{ha}^{-1}\right)$, contudo, as doses foram aplicadas no estádio V6.

Quanto à eficiência de uso de nitrogênio, os resutados foram superiores quando a adubação foi realizada no estádio V10, assemelhandos-se ao efeito do $\mathrm{N}$ na produtividade de grãos. Ao mesmo tempo, a eficiência agronômica, agrofisiológia e de recuperação descresceram à medida que as doses se elevaram, sendo mais positivo com a dose de $30 \mathrm{~kg} \mathrm{ha}^{-1} \mathrm{de} \mathrm{N}$, obtendo os valores de $16,70 \mathrm{~kg} \mathrm{~kg}^{-1}$, $17,33 \mathrm{~kg} \mathrm{~kg}^{-1}$ e $96,71 \%$, respectivamente, para as três avaliações (Tabela 2).

Estes resultados confirmam o já relatado por Fernandes et al. (2005), onde há diminuição da eficiência de uso de nitrogênio, de acordo com o aumento de doses de $\mathrm{N}$ aplicadas, em vista de o suprimento de nitrogênio exceder as necessidades da cultura.

Mesmo tratando-se de cultivo segunda safra, em que o clima é um dos fatores que interferem consideravemente no potencial da cultura, a eficiência de recuperação obtida de $96,71 \%$, na dose de $30 \mathrm{~kg} \mathrm{ha}^{-1}$ no estádio V10 deve ser ressaltada (Tabela 2), o que possivelmente minimizou as perdas do elemento para o ambiente, permitindo adequado acúmulo de $\mathrm{N}$ na parte aérea. De acordo com Rambo et al. (2007), a eficiência mundial estimada de uso do $\mathrm{N}$ em cereais é de apenas 33\%, sendo que uma das alternativas para aumentar esta eficiência é adotar melhores práticas de manejo, como o uso de dose e época de aplicação adequada.

Carvalho et al. (2012) concluíram que as cultivares de milho estudadas apresentaram características distintas quanto à eficiência no uso e absorção de N. Genótipos eficientes para o uso do nitrogênio e responsivos a sua aplicação podem ser selecionados para obter melhores resultados de produtividade.

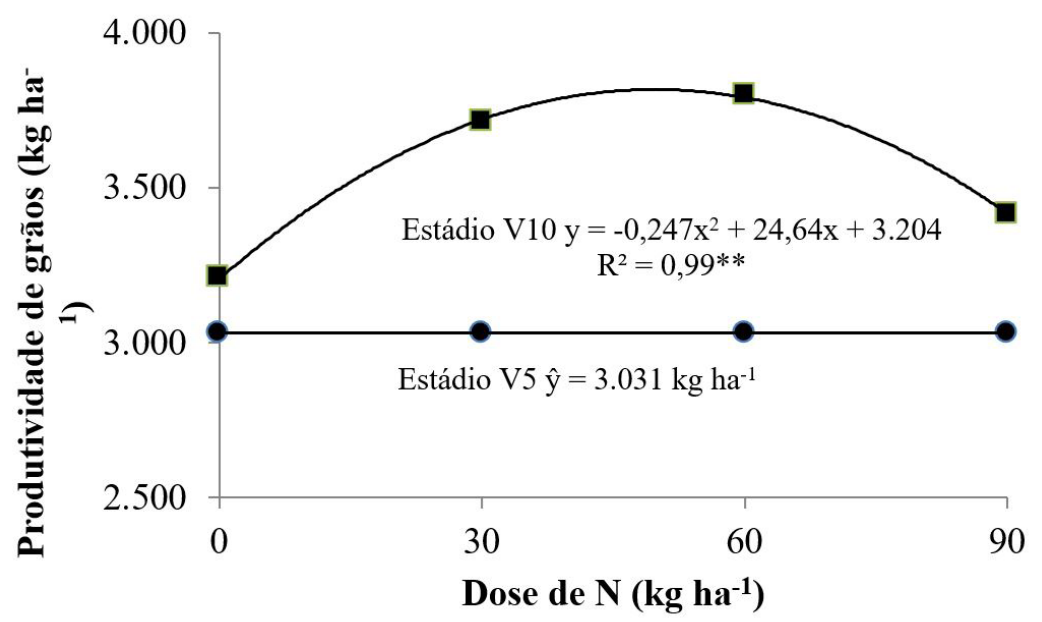

Figura 1. Interação significativa entre doses de $\mathrm{N}$ em cobertura e épocas de aplicação para a produtividade de grãos de milho safrinha. Barretos, SP, 2013. 
Tabela 2. Eficiência agronômica (EA), agrofisiológica (EAF) e de recuperação (ER) de uso de nitrogênio na cultura do milho safrinha em função de doses de $\mathrm{N}$ em cobertura e épocas de aplicação. Barretos, SP, 2013.

\begin{tabular}{|c|c|c|}
\hline \multirow{2}{*}{$\begin{array}{c}\text { Doses de } \mathrm{N} \text { em } \\
\text { cobertura }\end{array}$} & Estádio V5 & Estádio V10 \\
\hline & \multicolumn{2}{|c|}{ EA $\left(\mathrm{kg} \mathrm{kg}^{-1}\right)$} \\
\hline 0 & - & - \\
\hline 30 & 7,80 & 16,70 \\
\hline 60 & 2,36 & 9,90 \\
\hline \multirow[t]{2}{*}{90} & 3,50 & 3,30 \\
\hline & \multicolumn{2}{|c|}{ EAF $\left(\mathrm{kg} \mathrm{kg}^{-1}\right)$} \\
\hline 0 & - & - \\
\hline 30 & 3,79 & 17,33 \\
\hline 60 & 3,93 & 13,22 \\
\hline \multirow[t]{2}{*}{90} & 10,20 & 8,59 \\
\hline & \multicolumn{2}{|c|}{ ER (\%) } \\
\hline 0 & - & - \\
\hline 30 & 45,86 & 96,71 \\
\hline 60 & 43,20 & 56,57 \\
\hline 90 & 33,88 & 36,22 \\
\hline
\end{tabular}

A grande maioria dos trabalhos da cultura do milho sob influência da adubação nitrogenada em cobertura foi realizada nos estádios V4 ao V8, por tratar-se da fase de maior exigência, constituindo assim uma prática simples de reduzir perdas no ambiente. Entretanto, a resposta positiva com a aplicação no estádio V10, considerada uma época tardia, obtida no respectivo experimento, em contrário ao exposto acima, pode ocorrer em uma lavoura de milho safrinha, desde que não ocorra restrição hídrica para a solubilização do fertilizante e consequentemente a disponibilidade à planta. A falta de água no sistema não foi verificada durante a fase vegetativa da cultura no campo. A preocupação quanto aos riscos de perda da lavoura ou redução na produtividade na safrinha são relativamente grandes, sendo que um dos dilemas desse sistema de produção é saber a quantidade de $\mathrm{N}$ a ser aplicada, já que a deficiência hídrica altera a absorção e o metabolismo do $\mathrm{N}$ na planta, reduzindo a eficiência do fertilizante aplicado.

\section{Conclusões}

A adubação nitrogenada de cobertura promoveu acréscimos significativos no teor de $\mathrm{N}$ foliar e no número de grãos por espiga.

A produtividade foi superior com a aplicação de $50 \mathrm{~kg} \mathrm{ha}^{-1} \mathrm{de} \mathrm{N}$, sendo significativa no estádio V10.
Melhores resultados para a eficiência agronômica, agrofisiológica e de recuperação foram obtidos na adubação realizada no estádio V10, com aplicação de $30 \mathrm{~kg} \mathrm{ha}^{-1}$ de $\mathrm{N}$.

\section{Referências}

Brasil. Ministério da Agricultura, Pecuária e Abastecimento. (2009). Determinação do grau de umidade. In Brasil (Ed.), Regras para análise de sementes (398 p.). Brasília: MAPA/ACS.

Cantarella, H., \& Duarte, A. P. (1997). Tabela de recomendação de adubação NPK para milho "safrinha" no Estado de São Paulo. In Anais do 40 Seminário sobre a Cultura do Milho "Safrinha" (pp. 65-70). Campinas: Centro de Desenvolvimento do Vale do Paranapanema, Instituto Agronômico.

Carvalho, R. P., Von Pinho, R. G., \& David, L. M. C. (2012). Eficiência de cultivares de milho na absorção e uso de nitrogênio em ambiente de casa de vegetação. Semina: Ciências Agrárias, 33(6), 2125-2136.

Coelho, A. M., França, G. E., Pitta, G. V. E., \& Alves, V. M. C. (2002). Cultivo do milho: diagnose foliar do estado nutricional da planta (Comunicado Técnico, No. 45, 5 p.). Sete Lagoas: Embrapa-CNPMS.

Cruz, J. C., Pereira, I. A., Fo., \& Silva, G. H. (2012). Milho - cultivares para 2011/2012. Recuperado em 12 de dezembro de 2012, de http: www.cnpms.embrapa.br

Duarte, A. P., \& Kappes, C. (2013). Adubação de sistemas produtivos de milho safrinha e soja $(10$ p.). Campinas: IAC.

Fageria, N. K., \& Baligar, V. C. (2005). Enhancing nitrogen use efficiency in crop plants. Advances in Agronomy, 88(1), 97-185.

Fancelli, A. L., \& Dourado, D., No. (2008). Produção de milho (2a ed.). Guaíba: Agropecuária.

Farinelli, R., \& Lemos, L. B. (2012). Nitrogênio em cobertura na cultura do milho em preparo convencional e plantio direto consolidados. Pesquisa Agropecuária Tropical, 42(1), 63-70.

Fernandes, F. C. S., Buzetti, S., Arf, O., \& Andrade, J.A. C. (2005). Doses, eficiência e uso de nitrogênio 
por seis cultivares de milho. Revista Brasileira de Milho e Sorgo, 4(2), 195-204.

Ferreira, D. F. (2011). Sisvar: a computer statistical analysis system. Ciência e Agrotecnologia, 35, 1039-1042.

Fuentes, L. F. G., Souza, L. C. F., Serra, A. P., Rech, J., \& Vitorino, A. C. T. (2018). Corn agronomic traits and recovery of nitrogen from fertilizer during crop season and off-season. Pesquisa Agropecuária Brasileira, 53(10), 1158-1166.

Gazola, D., Zucarelli, C., Silva, R. R., \& Fonseca, I. C. B. (2014). Aplicação foliar de aminoácidos e adubação nitrogenada de cobertura na cultura do milho safrinha. Revista Brasileira de Engenharia Agrícola e Ambiental, 18(7), 700-707.

Góes, R. J., Rodrigues, R. A. F., Arf, O., \& Vilela, R. G. (2012). Nitrogênio em cobertura para o milho (Zea mays L.) em sistema plantio direto na safrinha. Revista Brasileira de Milho e Sorgo, 11(2), 169-177.

Gott, M., Sichock, D., Aquino, L. A., Xavier, F. O., Santos, L. P. D., \& Aquino, R. F. B. A. (2014). Fontes e épocas de aplicação de nitrogênio no milho safrinha. Revista Brasileira de Milho e Sorgo, 13(1), 24-34.
Kappes, C., Carvalho, M. A. C., Yamashita, O. M., \& Silva, J. A. N. (2009). Influência do nitrogênio no desempenho produtivo do milho cultivado na segunda safra em sucessão à soja. Pesquisa Agropecuária Tropical, 39(3), 251-259.

Malavolta, E., Vitti, G. C., \& Oliveira, S. A. (1997). Avaliação do estado nutricional das plantas ( $2^{\mathrm{a}} \mathrm{ed}$., pp. 115-230). Piracicaba: Associação Brasileira para Pesquisa da Potassa e do Fosfato.

Rambo, L., Strieder, M. L., Sangoi, L., Bayer, C., \& Argenta, G. (2007). Monitoramento do nitrogênio na planta e no solo para predição da adubação nitrogenada em milho. Pesquisa Agropecuária Brasileira, 42(3), 407-417.

Sichocki, D., Fuga, C. A. G., Aquino, L. A., Rua, R. A. A., \& Nunes, P. H. M. P. (2014). Resposta do milho safrinha à doses de nitrogênio e de fósforo. Revista Brasileira de Milho e Sorgo, 13(1), 48-58.

Soratto, R. P., Costa, T. A. M., Fernandes, A. M., Pereira, M., \& Maruyama, W. I. (2012). Parcelamento de fontes alternativas de nitrogênio no milho safrinha em sucessão à soja. Científica , 40(2), 179-188.

Souza, J. A., Buzetti, S., Teixeira, M. C. M., Fo., Andreotti, M., Sá, M. E., \& Arf, O. (2011). Adubação nitrogenada na cultura do milho safrinha irrigado em plantio direto. Bragantia, 70(2), 447-454. 